\title{
[gw22-e0135] SAFETY AND EFFICACY OF EARLY APPLICATION OF TIROFIBAN IN STEMI PATIENTS UNDERGOING PRIMARY PERCUTANEOUS CORONARY INTERVENTION
}

Fu Xianghua, Wang Zhigang, Wang Yanbo, Wang Xuechao, Fan Weise, Gu Xinshun, Hao Guozhen, Jiang Yunfa The Second Hospital of Hebei Medical University, Hebei, China

10.1136/heartjnl-2011-300867.445

Objective To explore the safety and efficacy of early application of tirofiban in STEMI patients undergoing primary percutaneous coronary intervention (PCI).

Methods From September 2008 to march 2011, 157 STEMI patients who were preparing for PCI were enrolled into this study. All patients were randomly divided into early group (patients received tirofiban in emergency room) and late group (patients received tirofiban immediately before PCI in catheterisation laboratory). The patients in each group were divided into different subgroups after coronal artery angiography on the basis of characteristic of infarction related artery (IRA). The clinical information, the result of PCI, and the incidence of major cardiovascular adverse events (MACE) during the hospitalisation were compared between two subgroups.

Results There were no significant difference in percentage of TIMI3 flow of IRA before PCI between early and late LAD group ( $p>0.05$ ). However, TIMI 3 flow was significantly less frequent with late RCA group compared with that in early RCA group ( $p>0.05)$. After dilation with balloon, cTFC was significantly lower in early LAD group ( $p>0.05)$. In the RCA comparison group, early application of tirofiban was associated with lower cTFC in culprit vessel compared with late application of tirofiban $(30.00 \pm 6.32$ vs $36.36 \pm 8.83, p<0.05)$. There were no significant difference in the percentage of TMPG $2 / 3$ perfusion between early and late LAD group ( $p>0.05)$, and TMPG $2 / 3$ perfusion was significantly less frequent with late RCA group ( $74 \%$ vs $47 \%, p<0.05)$. The percentage of thrombus during PCI was significantly less frequent in both early LAD and RCA groups (both $p>0.05$ ). There were no significant difference in the percentage of no-flow and slow-flow between early and late LAD group during the PCI $(p>0.05)$, and percentage of no-flow and slow-flow was significantly lower in early group than in late group ( $6 \%$ vs $22 \%, \mathrm{p}<0.05)$. The cardiovascular events such as cardiac death, reinfarction, revascularisation of IRA and serious angina did not happened in both LAD and RCA comparison groups during the hospitalisation.

Conclusions Early application of tirofiban could improve the effect of primary PCI and could effectively limit the expansion of infarction area. Early application of tirofiban dose not increases the incidence of MACE during the hospitalisation. 